\title{
Two Generalized Lower Bounds for the Circumference
}

\author{
Mossine S. Koulakzian and Zhora G. Nikoghosyan \\ Institute for Informatics and Automation Problems of NAS RA \\ e-mail: mossine@hotmail.com,zhora@ipia.sci.am
}

\begin{abstract}
In 2013, the second author obtained two lower bounds for the length of a longest cycle $C$ in a graph $G$ in terms of the length of a longest path (a longest cycle) in $G-C$ and the minimum degree of $G$ (Zh.G. Nikoghosyan, "Advanced Lower Bounds for the Circumference", Graphs and Combinatorics 29, pp. 1531-1541, 2013). In this paper we present two analogous bounds based on the average of the first $i$ smallest degrees in $G-C$ for appropriate $i$ instead of the minimum degree.
\end{abstract}

Keywords: Circumference, Minimum degree, Degree sums.

\section{Introduction}

Let $c$ be the circumference - the length of a longest cycle of a graph $G$ and $\delta$ the minimum degree in $G$.

In this paper we present the following two results.

Theorem 1. Let $C$ be a longest cycle in a graph $G, \hat{p}$ the order of a longest path in $G-C$ and $\mu$ the average of the first $\hat{p}$ smallest degrees in $G-C$. Then

$$
c \geq(\hat{p}+1)(\mu-\hat{p}+1) .
$$

Theorem 2. Let $C$ be a longest cycle in a graph $G$, $\hat{c}$ the order of a longest cycle in $G-C$ and $\mu$ the average of the first $\hat{c}$ smallest degrees in $G-C$. Then

$$
c \geq(\hat{c}+1)(\mu-\hat{c}+1) .
$$

Observing that $\mu \geq \delta$ in Theorems 1 and 2, we obtain the original lower bounds [2] as immediate corollaries in terms of $\hat{p}, \hat{c}$ and $\delta$.

Theorem A [2]. Let $C$ be a longest cycle in a graph $G$ and $\hat{p}$ the order of a longest path in $G-C$. Then

$$
c \geq(\hat{p}+1)(\delta-\hat{p}+1) .
$$

Theorem B [2]. Let $C$ be a longest cycle in a graph $G$ and $\hat{c}$ the order of a longest path in $G-C$. Then

$$
c \geq(\hat{c}+1)(\delta-\hat{c}+1)
$$




\section{Definitions}

We use Bondy and Murty [1] for terminology and notation not defined here, and consider only finite undirected graphs without loops and multiple edges. The vertex set of a graph $G$ is denoted by $V(G)$ or just $V$; the set of edges by $E(G)$ or just $E$. For a subgraph $H$ of $G$ we also use $G-H$ short for $G-V(H)$, and $|H|$ short for $|V(H)|$.

Paths and cycles in $G$ can be considered as connected subgraphs of $G$, having a maximum degree 0,1 or 2 . The length of a path $P$ and of a cycle $Q$, denoted by $l(P)$ and $l(Q)$, is $|V(P)|-1$ and $|V(Q)|$, respectively. We denote $l(P)=-1$ and $l(Q)=0$ if and only if $V(P)=V(Q)=\emptyset$. A graph is said to be Hamiltonian if its longest cycle passes through all of its vertices. The vertices and edges in $G$ can be interpreted as cycles of lengths 1 and 2 , respectively.

An $(x, y)$-path is a path with end vertices $x$ and $y$. Given an $(x, y)$-path $L$ of $G$ we denote by $\vec{L}$ the path $L$ with an orientation from $x$ to $y$. If $u, v \in V(L)$ then $u \vec{L} v$ denotes the consecutive vertices on $\vec{L}$ from $u$ to $v$ in the direction specified by $\vec{L}$. The same vertices, in reverse order, are given by $v \overleftarrow{L} u$. For $L=x \vec{L} y$ and $u \in V(L)$, let $u^{+}(\vec{L})$ (or just $u^{+}$) denote the successor of $u(u \neq y)$ on $\vec{L}$ and $u^{-}$denote its predecessor $(u \neq x)$. If $A \subseteq V(L)-y$ and $B \subseteq V(L)-x$, then we denote $A^{+}=\left\{v^{+} \mid v \in A\right\}$ and $B^{-}=\left\{v^{-} \mid v \in B\right\}$. A similar notation is used for the cycles. If $Q$ is a cycle and $u \in V(Q)$, then $u \vec{Q} u=u$. For $v \in V$, put $N(v)=\{u \in V \mid u v \in E\}, d(v)=|N(v)|$ and $\delta=\min \{d(u) \mid u \in V\}$.

\section{3. $\quad$ Special Definitions}

For the remainder of this section, let a subgraph $F$ of a graph $G$ and a path (or a cycle) $\vec{M}$ in $G-F$ be fixed.

Definition 1. $(* i)$-minimality, $(* i)$-maximality.

We use the notions of $(* i)$-minimality and $(* i)$-maximaliy defined with respect to certain operations for $i=1,2, \ldots, 10$. They will be described in detail currently.

Definition 2. MF-extension; $\vec{T}(u) ; \dot{u} ; \ddot{u}$.

For each $u \in V(M)$, let $\vec{T}(u)=u \vec{T}(u) \ddot{u}$ be a path in $G$, having only $u$ in common with $V(M)$. If $V(T(u)) \cap V(T(v))=\emptyset$ and $V(T(u)) \subseteq V(G-F)$ for all distinct vertices $u, v \in V(M)$, then the forest $T$, defined by $\{T(u) \mid u \in V(M)\}$, is said to be $M F$-extension. If $\ddot{u} \neq u$ for some $u \in V(M)$, then we use $\dot{u}$ to denote $u^{+}(\vec{T}(u))$.

Definition 3. $\Phi_{u} ; \varphi(u) ; \Psi(u) ; \psi(u)$.

Let $T$ be an $M F$-extension. For each $u \in V(M)$, put

$$
\begin{array}{ll}
\Phi_{u}=N(\ddot{u}) \cap V(T), & \varphi_{u}=\left|\Phi_{u}\right|, \\
\Psi_{u}=N(\ddot{u}) \cap V(F), & \psi_{u}=\left|\Psi_{u}\right| .
\end{array}
$$

Definition 4. $U_{0} ; \bar{U}_{0} ; U_{1} ; U^{*}$. 
Let $T$ be an $M F$-extension. Put

$$
\begin{gathered}
U_{0}=\{u \in V(M) \mid u=\ddot{u}\} ; \quad \bar{U}_{0}=V(M)-U_{0}, \\
U^{*}=\left\{u \in \bar{U}_{0} \mid \Phi_{u} \subseteq V(T(u))\right\} ; \quad U_{1}=V(M)-\left(U_{0} \cup U^{*}\right) .
\end{gathered}
$$

Definition 5. Maximal MF-extension.

An $M F$-extension $T$ is said to be maximal if it is extremal with respect to the following operation: - if there exists an edge $\ddot{u} z$ such that $u \in V(M)$ and $z \notin V(T) \cup V(F)$, then replacing $T(u)$ by $u T(u) \ddot{u} z$, we obtain a new $M F$-extension $T^{\prime}$ with $\left|V\left(T^{\prime}\right)\right|>|V(T)|$.

Definition 6. $\left(U_{0}\right)$-minimal and $\left(U_{0}, U^{*}\right)$-minimal $M F$-extensions.

An $M F$-extension $T$ is said to be $\left(U_{0}\right)$-minimal, if it is chosen such that $U_{0}$ is $(* 6)$-minimal (see the proof of Theorem 1). A $\left(U_{0}\right)$-minimal $M F$-extension $T$ is said to be $\left(U_{0}, U^{*}\right)$-minimal if it is chosen such that $U^{*}$ is $(* 10)$-minimal (see the proof of Theorem 2 ).

Definition 7. $B_{u} ; B_{u}^{*} ; b_{u} ; b_{u}^{*}$.

Let $T$ be an $M F$-extension and $u \in V(M)$. Put $B_{u}=\left\{v \in U_{0} \mid v \dot{u} \in E\right\}$ and $b_{u}=\left|B_{u}\right|$. By the definition, $B_{u}=\emptyset$ for each $u \in U_{0}$. Furthermore, for each $u \in U_{0}$, let $B_{u}^{*}=\left\{v \in \bar{U}_{0} \mid u \dot{v} \in E\right\}$ and $\left|B_{u}^{*}\right|=b_{u}^{*}$.

\section{Preliminaries}

The proofs of the following lemmas can be find in [2].

Lemma 1. Let $C$ be a cycle in a graph $G$ and $P$ a path in $G-C$. Let $\vec{P}_{0}, \ldots, \vec{P}_{p}$ be pairwise disjoint paths in $G-C$ with $\vec{P}_{i}=v_{i} \vec{P}_{i} w_{i}(i=0,1, \ldots, p)$, having only $v_{0}, \ldots, v_{p}$ in common with $P$. Then either there is a cycle in $G$ longer than $C$ or

$$
|C| \geq \sum_{i=0}^{p}\left|Z_{i}\right|+\left|\bigcup_{i=0}^{p} Z_{i}\right|,
$$

where $Z_{i}=N\left(w_{i}\right) \cap V(C)(i=0,1, \ldots, p)$.

Lemma 2. Let $F$ be a subgraph of a graph $G$ and $R$ a longest cycle in $G-F$ with a $\left(U_{0}\right)$ minimal $R F$-extension $T$. Then either there is a cycle longer than $R$ or $l(R) \geq \varphi_{u}+b_{u}+1$ for each $u \in U_{1}$.

Lemma 3. Let $F$ be a subgraph of a graph $G$ and $P$ a path in $G-F$ with a $\left(U_{0}\right)$-minimal $P F$ extension $T$. Then either there is a path longer than $P$ or $l(P) \geq \varphi_{u}+b_{u}$ for each $u \in U_{1} \cup U^{*}$. 


\section{Proofs}

Proof of Theorem 1. Let $Q=u_{0} \ldots u_{q}$ be a path in $G-C$ with a $\left(U_{0}\right)$ - minimal $Q C$ extension T. Assume without loss of generality that $C$ is $(* 1-* 4)$-extremal, and $Q$ is $(* 7-* 9)$-extremal. Since $G$ is non-Hamiltonian, we have $q \geq 0$.

Claim 1. If $u \in U_{0}$ and $v \in \bar{U}_{0}$, then $\Phi_{u} \cap V(T(v)) \subseteq\{v, \dot{v}\}$.

Proof. Suppose otherwise. Let $z \in V(T(v))-\{v, \dot{v}\}$. Then, replacing $T(u)$ and $T(v)$ by $u z \vec{T}(v) \ddot{v}$ and $v \vec{T}(v) z^{-}$, respectively, we can form (denote this operation by $(* 6)$ ) a new $Q C$-extension, contradicting the $\left(U_{0}\right)$ - minimality of $T$.

Claim 2. If $u \in U_{0}$, then $\varphi_{u} \leq q+b_{u}^{*}$.

Proof. The proof follows immediately from Definitions 3, 7 and Claim 1.

Claim 3. If $u \in \bar{U}_{0}$, then $\varphi_{u} \leq q-b_{u}$.

Proof. Using Lemma 3 with the fact that $Q$ is $(* 7-* 9)$-extremal, we obtain $q \geq \varphi_{u}+b_{u}$ for each $u \in \bar{U}_{0}$, and the result follows.

Observing that

$$
\sum_{u \in U_{0}} b_{u}^{*}=\sum_{u \in \bar{U}_{0}} b_{u}
$$

(by the definition) and using Claims 2 and 3, we obtain

$$
\sum_{i=0}^{q} \varphi_{u_{i}} \leq q(q+1)+\sum_{u \in U_{0}} b_{u}^{*}-\sum_{u \in \bar{U}_{0}} b_{u}=q(q+1) .
$$

Suppose first that $\varphi_{u_{i}}+\psi_{u_{i}} \neq d\left(\ddot{u}_{i}\right)$ for some $i \in \overline{0, q}$. Then there exists an edge $\ddot{u} z$ such that $z \notin V(T) \cup V(C)$. Adding $\ddot{u} z$ to $T$ we obtain a new $Q C$-extension, contradicting the maximality of $T$ (Definition 5). Now let $\varphi_{u_{i}}+\psi_{u_{i}}=d\left(\ddot{u}_{i}\right)(i=0, \ldots, q)$. Then

$$
\sum_{i=0}^{q} \psi_{u_{i}}=\sum_{i=0}^{q} d\left(\ddot{u}_{i}\right)-\sum_{i=0}^{q} \varphi_{u_{i}} \geq \sum_{i=0}^{q} d\left(\ddot{u}_{i}\right)-q(q+1) .
$$

It follows, in particular, that

$$
\max _{i}\left\{\psi_{u_{i}}\right\} \geq \frac{1}{q+1} \sum_{i=0}^{q} \psi_{u_{i}} \geq \frac{1}{q+1} \sum_{i=0}^{q} d\left(\ddot{u}_{i}\right)-q .
$$

By Lemma 1,

$$
\begin{gathered}
c \geq \sum_{i=0}^{q} \psi_{u_{i}}+\max _{i}\left\{\psi_{u_{i}}\right\} \\
\geq(q+2)\left(\frac{1}{q+1} \sum_{i=0}^{q} d\left(\ddot{u}_{i}\right)-q\right) \geq(q+2)\left(\mu_{q}-q\right) .
\end{gathered}
$$

Proof of Theorem 2. Let $H=u_{1} \ldots u_{k} u_{1}$ be a cycle in $G-C$ with an $\left(U_{0}, U^{*}\right)$-minimal $H C$-extension $T$. Let $H$ be $(* 5)$-extremal. Put

$$
U_{1}^{*}=\left\{u \in U^{*} \mid \varphi_{u} \leq \frac{h}{2}\right\}, \quad U_{2}^{*}=\left\{u \in U^{*} \mid \varphi_{u} \geq \frac{h+1}{2}\right\} .
$$


Claim 1. If $u \in U_{0}$ and $v \in \bar{U}_{0}$, then $\Phi_{u} \cap V(T(v)) \subseteq\{v, \dot{v}\}$.

Proof. The proof is very similar to that of Claim 1 in Theorem 1.

Claim 2. If $u \in U_{0}$, then $\varphi_{u} \leq h-1+b_{u}^{*}$.

Proof. Immediate from Definitions 3, 7 and Claim 1.

Claim 3. If $u \in U_{1}$, then $\varphi_{u} \leq h-1-b_{u}$.

Proof. Since $H$ is (*5)-extremal, by Lemma $2, h \geq \varphi_{u}+b_{u}+1$ for each $u \in U_{1}$, and the result follows.

Claim 4. If $u \in U^{*}$, then $\varphi_{u} \leq h-1-b_{u}+\varphi_{u}-\frac{h}{2}$.

Proof. Since $H$ is $(* 5)$-extremal, by the standard arguments, $h \geq 2\left(b_{u}+1\right)$ for each $u \in U^{*}$, and the result follows immediately.

Claim 5. If $u \in U_{1}$, then $\varphi_{u} \leq h-1-b_{u}$.

Proof. Immediate from Claims 3 and 4.

If $U_{2}^{*}=\emptyset$, then by Claims 2 and $5, \sum_{u} \varphi_{u} \leq h(h-1)$. But then, as in Theorem 1 , $c \geq(h+1)\left(\lambda_{1}-h+1\right)$, where $\lambda_{1}=\frac{1}{h} \sum_{i=1}^{k} d\left(\ddot{u}_{i}\right) \geq \mu_{h}$. Now let $U_{2}^{*} \neq \emptyset$. Choose $v \in U_{2}^{*}$ such that

$$
\varphi_{v}=\max _{u \in U_{2}^{*}}\left\{\varphi_{u}\right\}
$$

Claim 6. If $u \in U_{2}^{*}$, then $\varphi_{u} \leq h-1-b_{u}+\varphi_{v}-\frac{h}{2}$.

Proof. Immediate from (1) and Claim 4. we get

Using Claims 2, 5, 6 and recalling that $\sum_{u \in U_{0}} b_{u}^{*}=\sum_{u \in \bar{U}_{0}} b_{u}$ and $\left|U_{0}\right|+\left|U_{1} \cup U_{1}^{*}\right|+\left|U_{2}^{*}\right|=h$,

$$
\sum_{u} \varphi_{u}=\sum_{u \in U_{0}} \varphi_{u}+\sum_{u \in U_{1} \cup U_{1}^{*}} \varphi_{u}+\sum_{u \in U_{2}^{*}} \varphi_{u} \leq h(h-1)+\left|U_{2}^{*}\right|\left(\varphi_{v}-\frac{h}{2}\right) .
$$

By Definition 3, $\Phi_{v} \subseteq V(T(v))$. Let $v_{1}, \ldots, v_{t}$ be the elements of $\Phi_{v}^{+}$, occurring on $\vec{T}(v)$ in a consecutive order with $v_{t}=\ddot{v}$. Clearly $t=\left|\Phi_{v}\right|=\varphi_{v}$. Put

$$
N\left(v_{i}\right) \cap V(T)=\Phi_{i}^{\prime}, \quad N\left(v_{i}\right) \cap V(C)=Z_{i}^{\prime} \quad(i=1, \ldots, t) .
$$

If $\Phi_{i}^{\prime} \cap(V(T)-V(T(v))) \neq \emptyset$ for some $i \in \overline{1, t}$, then replacing $T(v)$ by

$$
v \vec{T}(v) v_{i}^{-} \ddot{v} \overleftarrow{T}(v) v_{i}
$$

we form (denote this operation by $(* 10)$ ) a new $H C$-extension, contradicting the minimality of $\left|U^{*}\right|$. So, we can assume $\Phi_{i}^{\prime} \subseteq V(T(v))(i=1, \ldots, t)$. Assume w.l.o.g. that $\max _{i}\left|\Phi_{i}^{\prime}\right|=$ $\left|\Phi_{t}^{\prime}\right|=\varphi_{v}$. So,

$$
\max _{i}\left|\Phi_{i}^{\prime}\right|=\left|\Phi_{t}^{\prime}\right|=\left|\Phi_{v}\right|=\varphi_{v}=t
$$

Since $\psi_{u_{i}}=d\left(u_{i}\right)-\varphi_{u_{i}}(i=1, \ldots, h)$ and $\left|Z_{i}^{\prime}\right|=d\left(v_{i}\right)-\left|\Phi_{i}^{\prime}\right|(i=1, \ldots, t-1)$, we have

$$
\sum_{i=1}^{h} \psi_{u_{i}}+\sum_{i=1}^{t-1}\left|Z_{i}^{\prime}\right|=\sum_{i=1}^{h}\left(d\left(u_{i}\right)-\varphi_{u_{i}}\right)+\sum_{i=1}^{t-1}\left(d\left(v_{i}\right)-\left|\Phi_{i}^{\prime}\right|\right)
$$




$$
=\sum_{i=1}^{h} d\left(u_{i}\right)+\sum_{i=1}^{t-1} d\left(v_{i}\right)-\sum_{i=1}^{h} \varphi_{u_{i}}-\sum_{i=1}^{t-1}\left|\Phi_{i}^{\prime}\right|
$$

Put

$$
\lambda_{2}=\frac{1}{h+t-1}\left(\sum_{i=1}^{h} d\left(u_{i}\right)+\sum_{i=1}^{t-1} d\left(v_{i}\right)\right) \geq \lambda_{1} \geq \mu_{h}
$$

Case 1. $\left|U_{2}^{*}\right|=1$.

By (2), (4) and (5),

$$
\begin{gathered}
\sum_{i=1}^{h} \psi_{u_{i}}+\sum_{i=1}^{t-1}\left|Z_{i}^{\prime}\right| \geq(h+t-1) \lambda_{2}-h(h-1)-t+\frac{h}{2}-\sum_{i=1}^{t-1} t \\
=(h+t-1) \lambda_{2}-h^{2}-t^{2}+\frac{3 h}{2}
\end{gathered}
$$

It follows, in particular, that

$$
\max _{i}\left\{\psi_{u_{i}},\left|Z_{i}^{\prime}\right|\right\} \geq \lambda_{2}-\frac{h^{2}+t^{2}-\frac{3 h}{2}}{h+t-1} \geq \lambda_{2}-\frac{3 h}{2}+2 .
$$

If $\lambda_{2} \leq h-1$, then clearly $c \geq(h+1)\left(\lambda_{2}-h+1\right)$. Let $\lambda_{2} \geq h \geq t+1$. Applying Lemma 1 to $Q=\ddot{v} \overleftarrow{T}(v) v \vec{H} v^{-}$, we get

$$
\begin{gathered}
c \geq \sum_{i=1}^{h} \psi_{u_{i}}+\sum_{i=1}^{t-1}\left|Z_{i}^{\prime}\right|+\max _{i}\left\{\psi_{u_{i}},\left|Z_{i}^{\prime}\right|\right\} \\
\geq(h+1)\left(\lambda_{2}-h+1\right)+(t-1)\left(\lambda_{2}-t-1\right) \geq(h+1)\left(\lambda_{2}-h+1\right) .
\end{gathered}
$$

Case 2. $\left|U_{2}^{*}\right| \geq 2$.

Choose $w \in U_{2}^{*}-v$ such that $\varphi_{v} \geq \varphi_{w} \geq \varphi_{u}$ for each $u \in U_{2}^{*}-\{v, w\}$. Define $w_{i}, Z_{i}^{\prime \prime}, \Phi_{i}^{\prime \prime}$ $(i=1, \ldots, r)$ for $T(w)$ in the same way as $v_{i}, Z_{i}^{\prime}$ and $\Phi_{i}^{\prime}$ were defined for $T(v)$. As in $(4)$, we can assume w.l.o.g. that $\max _{i}\left|\Phi_{i}^{\prime \prime}\right|=\left|\Phi_{r}^{\prime \prime}\right|=\left|\Phi_{w}\right|=\varphi_{w}=r$. Clearly, $t+r=\varphi_{v}+\varphi_{w} \geq h+1$. Then

$$
\begin{gathered}
\sum_{i=1}^{t}\left|Z_{i}^{\prime}\right|+\sum_{i=1}^{r}\left|Z_{i}^{\prime \prime}\right|=\sum_{i=1}^{t}\left(d\left(v_{i}\right)-\left|\Phi_{i}^{\prime}\right|\right)+\sum_{i=1}^{r}\left(d\left(w_{i}\right)-\left|\Phi_{i}^{\prime \prime}\right|\right) \\
=\sum_{i=1}^{t} d\left(v_{i}\right)+\sum_{i=1}^{r} d\left(w_{i}\right)-\sum_{i=1}^{t}\left|\Phi_{i}^{\prime}\right|-\sum_{i=1}^{r}\left|\Phi_{i}^{\prime \prime}\right| \geq(t+r) \lambda_{3}-t^{2}-r^{2},
\end{gathered}
$$

where

$$
\lambda_{3}=\frac{1}{t+r}\left(\sum_{i=1}^{t} d\left(v_{i}\right)+\sum_{i=1}^{r} d\left(w_{i}\right)\right) \geq \mu_{h}
$$

In particular,

$$
\max _{i}\left\{\left|Z_{i}^{\prime}\right|,\left|Z_{i}^{\prime \prime}\right|\right\} \geq \lambda_{3}-\frac{t^{2}+r^{2}}{t+r}
$$

Applying Lemma 1 to $Q=\ddot{v} \overleftarrow{T}(v) v \vec{H} w \vec{T}(w) \ddot{w}$, we get

$$
c \geq \sum_{i=1}^{t}\left|Z_{i}^{\prime}\right|+\sum_{i=1}^{r}\left|Z_{i}^{\prime \prime}\right|+\max _{i}\left\{\left|Z_{i}^{\prime}\right|,\left|Z_{i}^{\prime \prime}\right|\right\} \geq(t+r) \lambda_{3}-t^{2}-r^{2}+\lambda_{3}-\frac{t^{2}+r^{2}}{t+r}
$$




$$
\geq(h+1)\left(\lambda_{3}-h+1\right)+\lambda_{3}(t+r-h)+h^{2}-1-t^{2}-r^{2}-\frac{t^{2}+r^{2}}{t+r} .
$$

If $\lambda_{3} \leq h-1$, then clearly, $c \geq(h+1)\left(\lambda_{3}-h+1\right)$. Otherwise,

$$
\begin{gathered}
c \geq(h+1)\left(\lambda_{3}-h+1\right)+h(t+r)-1-t^{2}-r^{2}-\frac{t^{2}+r^{2}}{t+r} \\
\geq(h+1)\left(\lambda_{3}-h+1\right)+(h-1)(t+r)-t^{2}-r^{2} .
\end{gathered}
$$

Observing that

$$
h-1 \geq \max \{t, r\} \geq \frac{t^{2}+r^{2}}{t+r},
$$

we obtain $c \geq(h+1)\left(\lambda_{3}-h+1\right)$. Thus, $c \geq(h+1)(\lambda-h+1)$, where $\lambda=\min \left\{\lambda_{1}, \lambda_{2}, \lambda_{3}\right\} \geq \mu_{h}$.

\title{
References
}

[1] J.A. Bondy and U.S.R. Murty, Graph Theory with Applications. Macmillan, London and Elsevier, New York, 1976.

[2] Zh.G. Nikoghosyan, "Advanced lower bounds for the circumference", Graphs and Combinatorics 29, pp. 1531-1541, 2013.

Submitted 22.08.2017, accepted 24.12.2017.

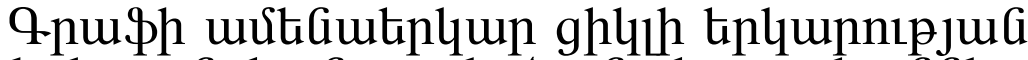

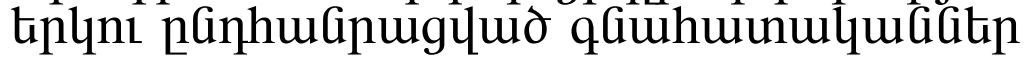

\author{
U. enıцupquua la $\sigma$. Uhlnnnujua
}

\section{Uরựnนnıu}

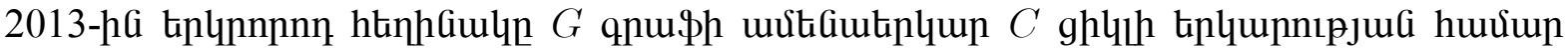

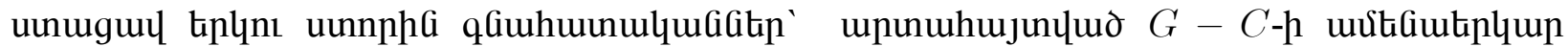

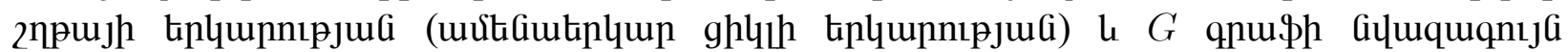
uuunfough pGnıpuqnh_Gipny (Zh.G. Nikoghosyan, Advanced Lower Bounds for the Circumference, Graphs and Combinatorics 29, pp. 1531-1541, 2013): Gtplqu u2fuuunuGpnuर

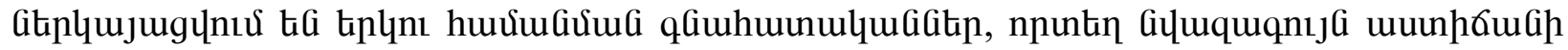

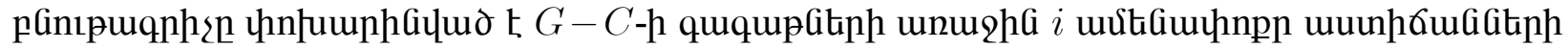

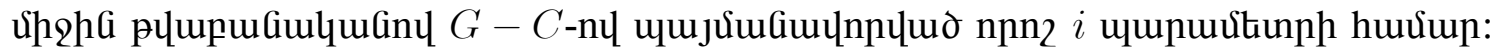




\title{
Аве обобщенные нижние оценки Аля длины Алиннейшего цикла графа
}

М. Кулакзян и Ж. Никогосян

\begin{abstract}
Аннотация
В 2013 году второй автор получил две нижние оценки для длины длиннейшего цикла графа $G$ выраженные через Алину Алиннейшей цепи (Алиннейшего цикла) подграфра $G-C$ и минимальную степень графра $G$ (Zh.G. Nikoghosyan, Advanced Lower Bounds for the Circumference, Graphs and Combinatorics 29, pp. 1531-1541, 2013)? В настоящей работе представляются две обобщенные аналогичные оценки, где вместо минимальной степени рассматривается средняя арифрметическая степеней первых $i$ наименьших степеней вершин подграфа $G-C$ для подходящего параметра $i$.
\end{abstract}

